\title{
Pancreatic pseudocyst-portal vein fistula with refractory hepatic pseudocyst: Two cases treated with EUS cyst-gastrostomy and review of the literature
}

다(1)우우

\author{
Authors \\ John Eccles ${ }^{1}$, Edward Wiebe², Pernilla D'Souza', Gurpal Sandha'
}

Institutions

1 Division of Gastroenterology, University of Alberta Hospital, Edmonton, Alberta, Canada

2 Department of Diagnostic Imaging, University of Alberta Hospital, Edmonton, Alberta, Canada

submitted 3.5.2018

accepted after revision 2.9.2018

Bibliography

DOI https://doi.org/10.1055/a-0754-2247 |

Endoscopy International Open 2019; 07: E83-E86

(c) Georg Thieme Verlag KG Stuttgart · New York ISSN 2364-3722

Corresponding author

Dr. Gurpal Sandha, MBBS, FRCPC, Professor of Medicine,

Division of Gastroenterology, University of Alberta Hospital, 2-76 Zeidler-Ledcor Centre, 8540-112 Street, Edmonton, Alberta, T6G 2X8 Canada

Phone: +780-492-8170

Fax: +780-492-1699

gsandha@ualberta.ca

\section{ABSTRACT}

Background and study aims Pseudocysts are the most common pancreatic cystic lesions and they usually develop in association with pancreatitis of at least 4 weeks' duration. Extra-pancreatic pseudocysts, although reported, are relatively uncommon. Secondary liver pseudocysts are recognized within the literature, and most patients described have required percutaneous or surgical drainage due to infection or symptoms. The mechanism of hepatic pseudocyst formation is not entirely clear but it is postulated that this phenomenon may occur through pseudocyst-portal vein fistulization. We describe two cases of patients presenting with pancreatic pseudocysts invading the portal venous system with embolization of pancreatic fluid to the liver and subsequent hepatic pseudocyst formation. Interestingly, liver pseudocyst resolution was incomplete with antibiotics and percutaneous drainage alone, and only occurred following endoscopic ultrasonography-guided pancreatic cyst-gastrostomy and metal stent insertion. We have reviewed the current literature on the diagnosis and management of pseudocyst-portal vein fistula formation and we believe that our cases represent the first published within the literature to describe this treatment approach.

\section{Introduction}

Pancreatic pseudocyst-portal vein fistulization is a rarely described phenomenon within the literature [1-6]. We present two cases of patients with pancreatic pseudocysts fistulizing into the portal venous system and seeding to the liver with subsequent hepatic pseudocyst formation. Complete and longterm resolution of the liver pseudocysts failed with more conservative measures, including parenteral antibiotics and percutaneous drainage, and was achieved only after endoscopic ultrasonography (EUS)-guided pancreatic cyst-gastrostomy and metal stent insertion. We have reviewed the current literature on diagnosis and management of hepatic pseudocysts and pseudocyst-portal vein fistulization.

\section{Case reports}

\section{Patient 1}

A 64-year-old alcoholic man presenting with abdominal pain, weight loss and anorexia, underwent abdominal computed tomography $(\mathrm{CT})$, which revealed a $3.6 \times 2 \mathrm{~cm}$ pancreatic cyst, portal vein thrombosis and a multiloculated, septated and hypoattenuating liver lesion within segment eight, measuring $6.7 \times 5.6 \mathrm{~cm}$. Linear EUS confirmed diffuse calcification throughout the pancreas, but no pancreatic duct dilatation, and a $4.5-\mathrm{cm}$ pancreatic head pseudocyst. Percutaneous fineneedle aspiration (FNA) of the liver cyst yielded serosanguinous fluid. The patient was treated with antibiotics and percutaneous drainage of presumed liver abscess and discharged, but he presented 3 months later with abdominal pain. At that time, abdominal CT showed enlargement of the liver cyst, which measured $7.6 \times 7.2 \mathrm{~cm}$ in diameter and a significant increase in size of 


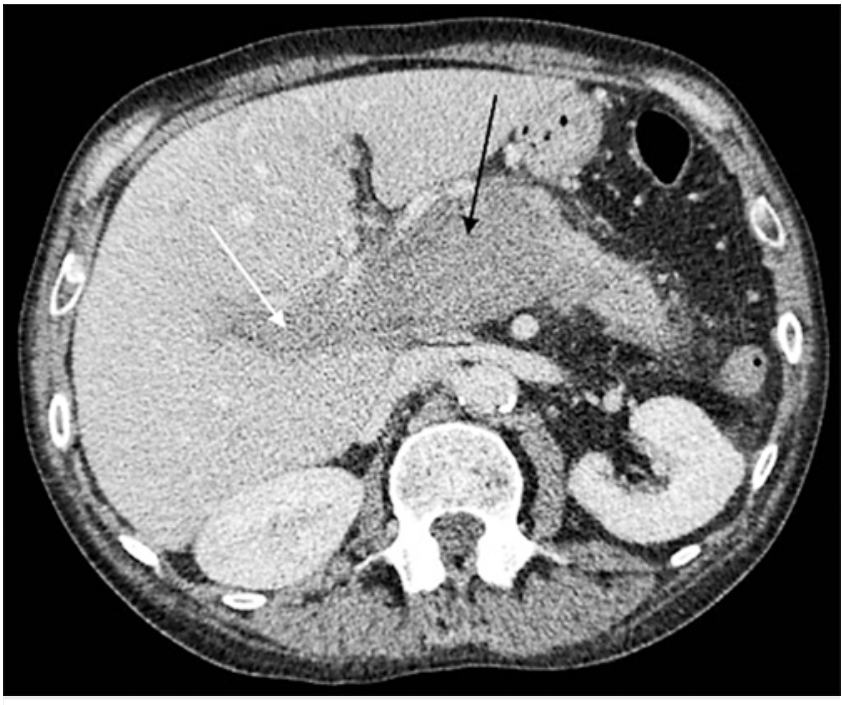

- Fig. 1 Axial CT image of pancreatic pseudocyst (black arrow) with extension to portal venous system (white arrow).

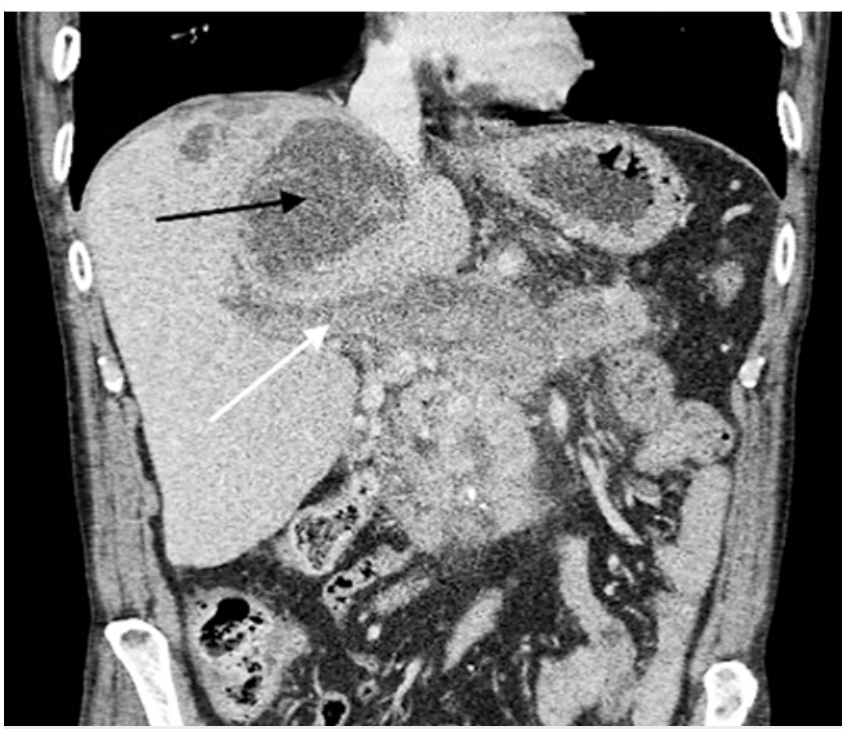

- Fig. 2 Coronal image of liver pseudocyst (black arrow) and hyperdense fluid in portal vein (white arrow) and pancreatic pseudocyst.

the pancreatic pseudocyst, now $8.2 \times 4.8 \mathrm{~cm}$ and communicating with the pancreatic duct. Interestingly, hyperdense material was seen extending from the pseudocyst into the portal vein ( $\triangleright$ Fig. 1 and $\triangleright$ Fig. 2). Further FNA of the liver cyst demonstrated fluid rich in amylase and lipase (1950 U/L and 3389U/L, respectively), suggesting a pancreatic origin. Despite CT-guided percutaneous drainage of the pancreatic pseudocyst, follow-up imaging several weeks later revealed an increase in its size. Endoscopic retrograde pancreatography (ERP) demonstrated an abrupt cut-off approximately $2 \mathrm{~cm}$ into the pancreatic duct, but with no evidence of communication with a cystic cavity. Given the documented increase in size of the pseudocyst, another linear EUS was performed, confirming a $5 \times 5.5 \mathrm{~cm}$ hypoechoic lesion adjacent to the posterior stomach wall. Murky brown fluid

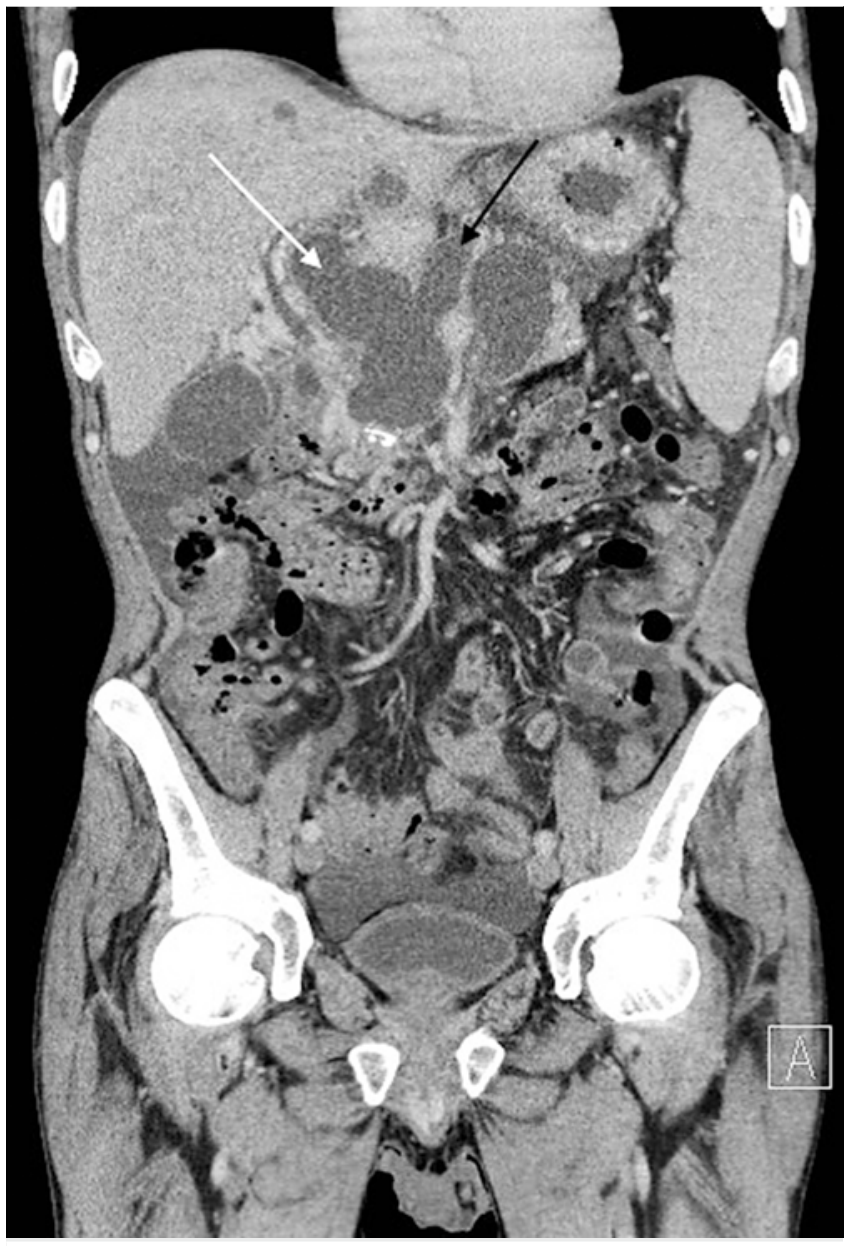

- Fig. 3 Coronal CT image shows hypodense fluid in pancreatic pseudocyst (black arrow) communicating with the main portal vein (white arrow).

was aspirated and sent for culture and an EUS-guided cyst-gastrostomy was performed with placement of two 7-Fr plastic double-pigtail stents within the pseudocyst. Repeat abdominal CT documented a significant decrease in size of both the pancreatic pseudocyst and the intrahepatic fluid collection. The patient was discharged home on oral antibiotics. On follow-up CT 2 months later, however, the pancreatic pseudocyst had grown again to $7.4 \mathrm{~cm}$ in diameter. The patient subsequently underwent another EUS-guided cyst-gastrostomy with removal of the plastic stents and insertion of a 4-cm fully-covered self-expanding metal stent (FCSEMS). A follow-up abdominal CT 2 months later showed almost complete resolution of both the liver and pancreatic collections. The patient has not required any further admissions to the hospital.

\section{Patient 2}

In a 54-year-old man presenting with abdominal pain and weight loss, abdominal CT identified similar features of chronic pancreatitis and an ill-defined, multiloculated, hypoattenuating liver mass in segments 8 and $4 \mathrm{~A}$, measuring $6.8 \times 8.8 \mathrm{~cm}$. Extensive occlusive thrombus was also seen involving the portal, superior mesenteric and splenic veins. Percutaneous aspira- 


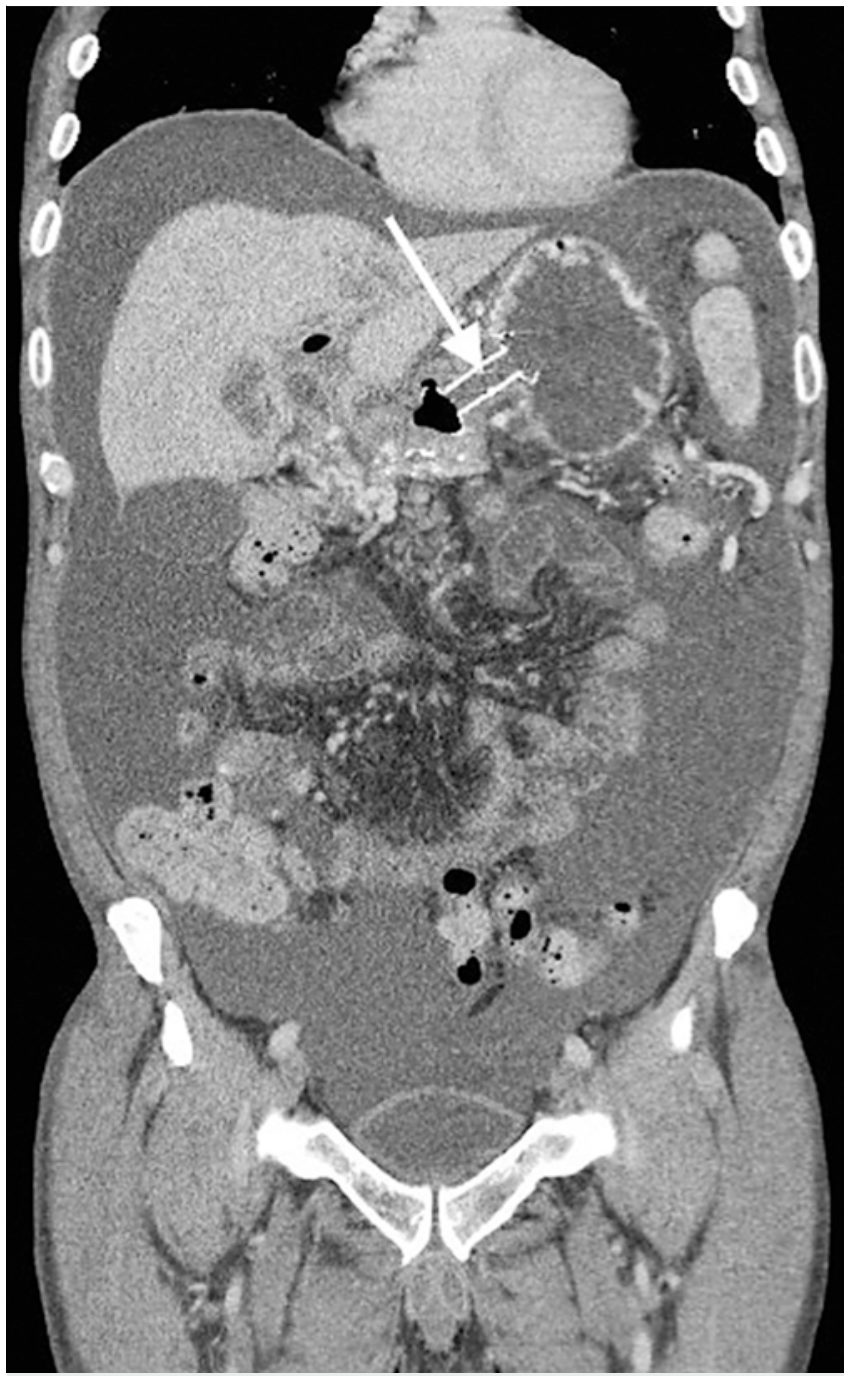

- Fig. 4 Coronal image showing cyst-gastrostomy stent (white arrow) in place with drainage of the pancreatic pseudocyst and portal venous system.

tion of the liver mass suggested an abscess, prompting treatment with intravenous (IV) antibiotics and drainage. Three months later, the patient presented with abdominal pain and ascites. Repeat abdominal CT showed evidence of complex fluid within the portal venous system, suspicious for a pancreatic pseudocyst origin ( $\triangleright$ Fig.3). ERP demonstrated communication between a dilated pancreatic duct and an irregular tubularshaped structure, consistent with a pseudocyst. A 7-Fr plastic pancreatic stent was inserted. Follow-up magnetic resonance cholangiopancreatography (MRCP) confirmed complete distal migration of the pancreatic stent and a pancreatic ductal stricture with decompression into a pseudocyst and evidence of secondary cavernous transformation of the portal vein extending into the liver. Percutaneous aspiration of the large liver cyst confirmed fluid rich in amylase (>2000 U/L). The patient remained hospitalized on IV antibiotics and a further attempt at ERP and pancreatic duct stenting was unsuccessful. Because the man was not felt to be a suitable surgical candidate, we pro- ceeded with linear EUS-guided cyst-gastrostomy and deployment of a 4-cm FCSEMS. Significant bleeding at the time of stent deployment led to urgent angiography, however, no active bleeding point was identified, and the patient was managed conservatively. Abdominal CT 2 weeks later showed successful decompression of the pancreatic pseudocyst and resolving hepatic pseudocyst ( Fig.4).

\section{Discussion}

Pancreatic pseudocysts can present various complications, some more common such as infection, rupture, and gastrointestinal obstruction, to the slightly rarer. Hepatic pseudocysts are a recognized but rare phenomenon described within published case reports [7-12]. In one report of a patient presenting with a large liver cystic lesion in association with a pancreatic tail pseudocyst, the authors proposed several potential mechanisms for development of hepatic pseudocysts, including through direct invasion or possibly presence of heterotopic pancreatic tissue within the liver. Interestingly, they also reported a $90 \%$ cure rate seen with percutaneous drainage, subsequently recommended to be considered the first-line treatment. Interestingly, our two cases did not demonstrate successful resolution following this approach [7].

Having reviewed the literature, there are very few published reports of liver pseudocyst development as a direct consequence of invasion of the portal vein, or "fistulization" of a pancreatic pseudocyst [2-6]. In one case report outlining development of a pancreatic pseudocyst-portal vein fistula and thrombosis, the authors postulated the mechanism of a "high-pressure system" within the pseudocyst promoting deposition of pancreatic fluid material into the portal vein. In their case, percutaneous liver abscess drainage and insertion of a plastic pancreatic stent across a proximal pancreatic duct stricture led to improvement [4].

Another case report describes a patient presenting with a large pancreatic pseudocyst adjacent to a dilated, low-density fluid-filled portal vein and liver abscess. Percutaneous transhepatic portography confirmed the diagnosis of a pseudocystportal vein fistula. At ERP, the patient had evidence of a pancreatic duct stricture and communication with the pseudocyst, and pancreatic duct stenting was successful in achieving resolution of both pancreatic and hepatic fluid collections [3].

One report describes several cases of pseudocyst-portal vein fistulization, the authors postulating that portal vein thrombosis occurs in these patients as a consequence of rather than encouraging development of the fistula. In their discussion, they suggest a strong relationship between alcohol excess and development of pseudocyst-portal vein fistula, particularly in association with chronic pancreatitis. CT may be limited in correctly diagnosing pseudocyst-portal vein fistula, findings often initially suggesting only portal vein thrombosis, although in our cases, pseudocyst-portal vein fistula development in association with portal vein thrombosis was recognized on review of the images by a highly-experienced radiologist [6].

In another case report, the authors refer to 18 other cases of pseudocyst-portal vein fistulization; nine had been treated sup- 
portively, six required surgical intervention, such as pancreaticoenterostomy, and three improved with endoscopic pancreatic duct stenting. Mortality occurred in five of 18 patients, likely due to massive release of pancreatic enzymes into the portal circulation and resulting systemic lipolysis. While they describe a role for surgical cyst-gastrostomy in pseudocyst-portal vein fistula, this had failed to prevent portal vein rupture and death in their patient. The authors propose that erosion from mass effect of the pseudocyst and release of digestive enzymes into the portal vein may lead to development of intravascular thrombosis. They recommend that patients with portal vein thrombosis in the setting of a pseudocyst-portal vein fistula be considered for pancreatic duct stenting and percutaneous pseudocyst drainage [5].

Generally, most described cases of hepatic pseudocysts within case reports were managed conservatively or drained surgically or percutaneously. Our cases are among the first to demonstrate that secondary or "seeded" liver pseudocysts, refractory to percutaneous drainage and antibiotics, resolve completely after decompression of their feeding "primary" pancreatic pseudocyst through EUS-guided cyst-gastrostomy and metal stenting. Failure of percutaneous drainage alone in treating hepatic pseudocysts might be explained by persistent feeding with proteolytic material via the connection between the primary pseudocyst and portal vein. Interestingly, both of our patients demonstrated pancreatic ductal strictures at ERP, but only one underwent pancreatic duct stenting, later found to have migrated, and the other was not amenable to stenting. Despite this, each patient showed complete pancreatic and hepatic pseudocyst resolution with endoscopic cyst-gastrostomy and metal stent placement alone.

\section{Conclusion}

In conclusion, hepatic pseudocysts may develop in association with pancreatic pseudocysts through an "embolic" phenomenon mediated through the portal venous system. Furthermore, failure of liver cyst resolution could be attributed to ongoing "feeding" from its parent pancreatic pseudocyst. We propose that primarily treating hepatic pseudocysts without targeting the pancreatic pseudocyst in this setting will result in failure of complete resolution and therefore EUS-guided pancreatic cystgastrostomy and metal stent placement should be strongly considered as first-line therapy to achieve decompression in pancreatic pseudocyst-portal vein fistula with secondary he- patic pseudocyst, or second-line therapy in those who do not improve with endoscopic pancreatic duct stenting.

\section{Competing interests}

GS is a consultant for Boston Scientific Corp. and has received honoraria for speaking engagements. JE, EW and PDS declare no conflict relevant to this manuscript.

References

[1] Byrne MF, Baillie J. Pancreatic Fluid Collections and Pseudocysts in Patients with Acute Pancreatitis. In: Forsmark CE, ed. Pancreatitis and Its Complications. 1st ed. Totowa, New Jersey: Humana Press Inc; 2005

[2] Skarsgard ED, Ellison E, Quenville N. Spontaneous rupture of a pancreatic pseudocyst into the portal vein. Can J Surg 1995; 38: 459-463

[3] Noh R, Kim HJ. A pancreatic pseudocyst-portal vein fistula closed by endoscopic pancreatic stent insertion. Gastrointestinal Endoscopy 2010; 72: $1103-1105$

[4] Raza SS, Hakeem A, Sheridan M et al. Spontaneous pancreatic pseudocyst-portal vein fistula: a rare and potentially life-threatening complication of pancreatitis. Ann R Coll Surg Engl 2013; 95: e7 - e9

[5] Ng TS, Rochefort H, Czaplicki C et al. Massive pancreatic pseudocyst with portal vein fistula: Case report and proposed treatment algorithm. Pancreatology 2015; 15: 88-93

[6] Alessandrino F, Strickland C, Mojtahed A et al. Clinical and cross-sectional imaging features of spontaneous pancreatic pseudocyst-portal vein fistula. Clinical Imaging 2017; 44: 22 - 26

[7] Mofredj A, Cadranel JF, Dautreaux M et al. Pancreatic Pseudocyst Located in the Liver: A Case Report and Literature Review. J Clin Gastroenterol 2000; 30: 81-83

[8] Les I, Córdoba J, Vargas V et al. Pancreatic Pseudocyst located in the liver. Rev Esp Enferm Dig 2006; 98: 616-620

[9] Guesmi F, Zoghlami A, Saidi Y et al. Pancreatic pseudocysts located in the liver: A systematic review of the literature. Tunis Med 2009; 87: $801-804$

[10] Martínez Sanz N, González-Valverde FM, Vicente-Ruiz M el al. Intrahepatic pancreatic pseudocyst: a case report. Rev Esp Enferm Dig 2015; 107: $249-250$

[11] Topno N, Ghosh S, Baruah A. a rare case report of hepatic subcapsular pseudocyst of pancreas. J Clin Diagnos Res 2016; 10: $18-19$

[12] Edmonds P, Arroyo JP, Morales A. Intrahepatic pancreatic pseudocyst: a rare complication of pancreatitis. J Gastrointestin Liver Dis 2017; 26: 222 